LA-UR-99- $\quad 2181$

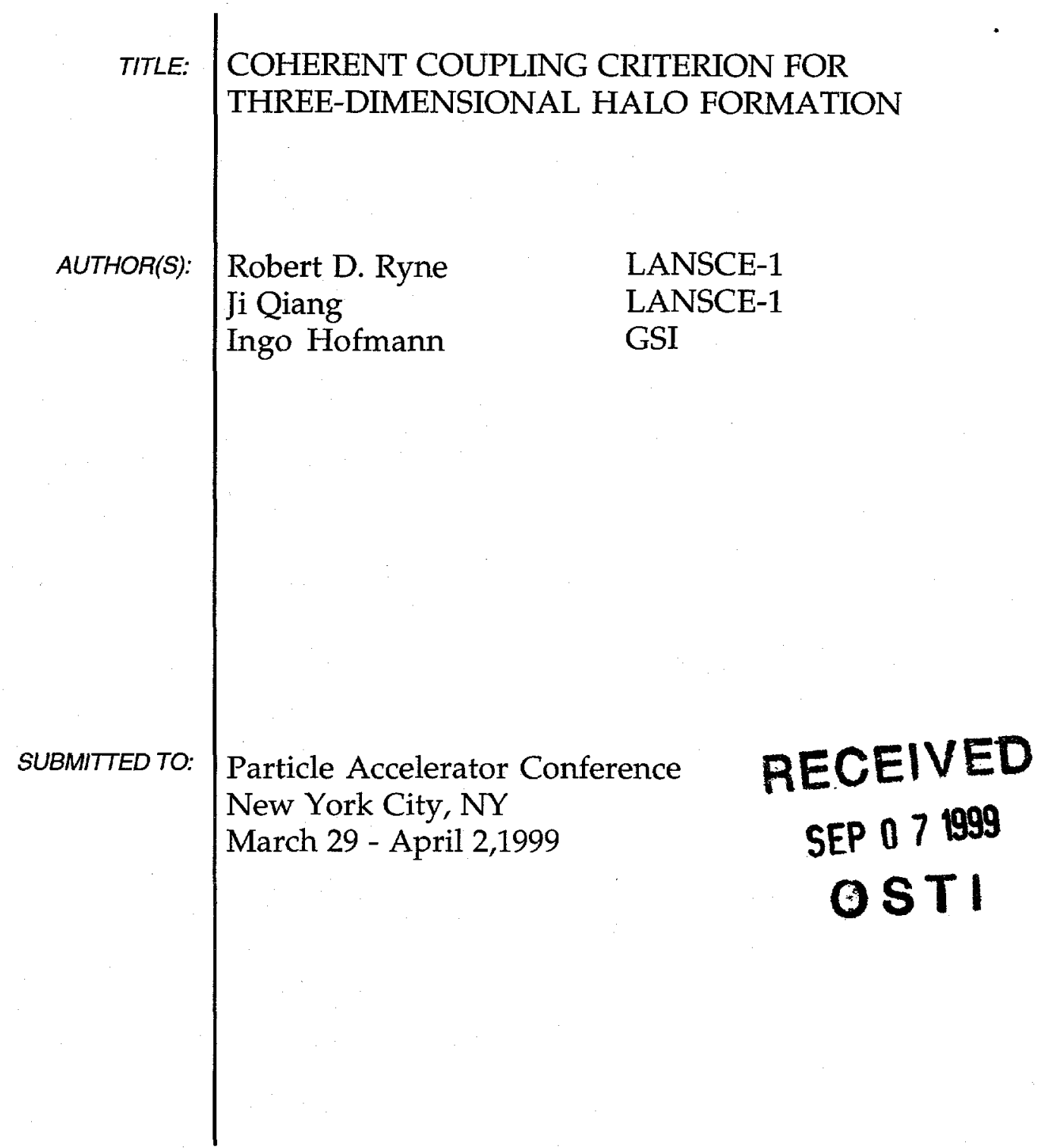

Los
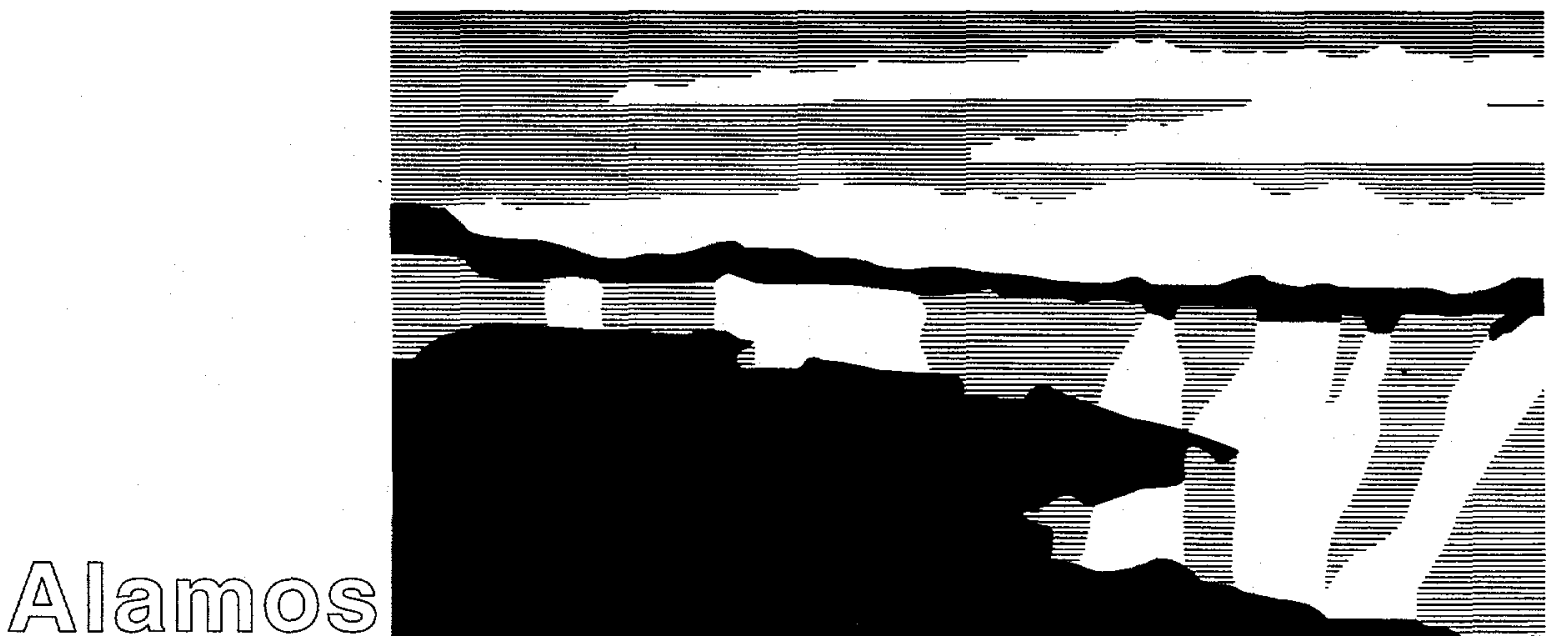

NATIONAL LABORATORY

Los Alamos National Laboratory, an affirmative action/equal opportunity employer, is operated by the University of California for the U.S. Department of Energy under contract W-7405-ENG-36. By acceptance of this article, the publisher recognizes that the U.S. Government retains a nonexclusive, royalty-free license to publish or reproduce the published form of this contribution, or to allow others to do so, for U.S. Government purposes. The Los Alamos National Laboratory requests that the publisher identify this article as work performed under the auspices of the U.S. Department of Energy. 


\section{DISCLAIMER}

This report was prepared as an account of work sponsored by an agency of the United States Government. Neither the United States Government nor any agency thereof, nor any of their employees, make any warranty, express or implied, or assumes any legal liability or responsibility for the accuracy, completeness, or usefulness of any information, apparatus, product, or process disclosed, or represents that its use would not infringe privately owned rights. Reference herein to any specific commercial product, process, or service by trade name, trademark, manufacturer, or otherwise does not necessarily constitute or imply its endorsement, recommendation, or favoring by the United States Government or any agency thereof. The views and opinions of authors expressed herein do not necessarily state or reflect those of the United States Government or any agency thereof. 


\section{DISCLAIMER}

Portions of this document may be illegible in electronic image products. Images are produced from the best available original document. 


\title{
Coherent Coupling Criterion for Three-Dimensional Halo Formation
}

\author{
I. Hofmann, GSI, Ji Qiang, R. Ryne, LANL
}

\section{Abstract}

In this paper we study coupling between the transverse and longitudinal degrees-of-freedom in intense, rms mismatched charged particle beams. We find that a coherent ( $2: 1$, i.e. parametric) resonance beween the transverse and longitudinal mismatch eigenmodes has the effect that a transverse "breathing mode" mismatch can excite a longitudinal mismatch and halo. If the resonance condition is not satisfied we find practically no coupling. We compare results obtained with the $3 \mathrm{D} \mathrm{rms}$ envelope equations in a uniform focusing channel with those obtained using large scale, 3D parallel Particle-In-Cell (PIC) simulations.

\section{INTRODUCTION}

The study of halos in beams for new high intensity accelerator applications has so far focused mainly on twodimensional models of the transverse halo. In recent work on three-dimensional effects the presence of coupling phenomena was recognized for a special class of fully selfconsistent equipartitioned equilibria, where it was claimed that longitudinal or transverse halo results for small mismatch (10\%) if the mismatch in the other plane is large [1].

In this paper we examine the coupling issue using the 3D ms envelope equations and PIC simulations. Unlike Ref. [1] we allow our beams to be non-equipartitioned. In practical linac design the requirement of strict equipartitioning [2] appears to be unnecessarily stringent; a recent study of anisotropy effects showed that "temperature" ratios of 2-3 between different degrees of freedom do not necessarily give rise to instability and energy exchange [3]. We therefore investigate these more general anisotropic equilibria subject to rms mismatch. The matched bunches are not equilibria in a strict sense (i.e. functions of the Hamiltonian), but our simulations show - confirming the $2 \mathrm{D}$ analysis of Ref. [3] - that within the constraint of modest anisotropy the rms quantities remain practically constant.

\section{ENVELOPE MODEL}

For simplicity we assume constant focusing and bunched beams with a rotational axis, which restricts the analysis to the transverse "breathing mode" [4] generalized by the presence of longitudinal motion, as well as a longitudinal mode.The rms envelope equations can be written as

$$
\begin{array}{r}
\frac{d^{2} a}{d t^{2}}+k_{0, x}^{2} a-\frac{3 N r_{c}}{2 \beta^{2} \gamma^{3} a c}\left(1-f / \gamma^{2}\right)-\frac{\epsilon_{x}^{2}}{a^{3}}=0 \\
\frac{d^{2} c}{d t^{2}}+k_{0, z}^{2} c-\frac{3 N r_{c}}{\beta^{2} \gamma^{5} a^{2}} f-\frac{\epsilon_{z}^{2}}{c^{3}}=0
\end{array}
$$

The geometry factor $f$ depends on the aspect ratio $p \equiv c / a$,

$$
f \equiv \frac{p \operatorname{arccosh}(p)}{\left(p^{2}-1\right)^{3 / 2}}-\frac{1}{\left(p^{2}-1\right)} \quad(p>1)
$$

with $p^{2}-1$ replaced by $1-p^{2}$ for $p<1$. The transverse "quadrupolar" mode may be included, if desired, using separate equations for $x$ and $y$ and a more general form of $f$.

For matched beams we assume vanishing time derivatives. The resulting algebraic equations determine the $\mathrm{ms}$ matching conditions, which are also used to initialize the PIC simulations. It is convenient to introduce the space charge shifted wavenumbers $k_{x, z}$ according to

$$
\begin{array}{r}
k_{x}^{2}=k_{0, x}^{2}-\frac{3 N r_{c}}{2 \beta^{2} \gamma^{3} a^{2} c}\left(1-f / \gamma^{2}\right) \\
k_{z}^{2}=k_{0, z}^{2}-\frac{3 N r_{c}}{\beta^{2} \gamma^{5} a^{2} c} f
\end{array}
$$

For small perturbations the rms equations can be linearized about the matched values $c_{0}, a_{0}$, which yields the two eigenmodes of coupled envelope oscillation with corresponding eigenfrequencies $\omega_{t r, l o}$. For vanishing space charge the "transverse" eigenmode oscillates with $2 k_{0, x}$, and the "longitudinal" eigenmode with $2 k_{0, z}$. As is typical for systems with two degrees of freedom, results can be represented in terms of three dimensionless parameters which can be chosen conveniently. In Fig. 1 we show the envelope frequencies for fixed $c_{0} / a_{0}=1.5$ and $k_{0, z} / k_{0, x}=0.6$. Note that the space charge limit is reached

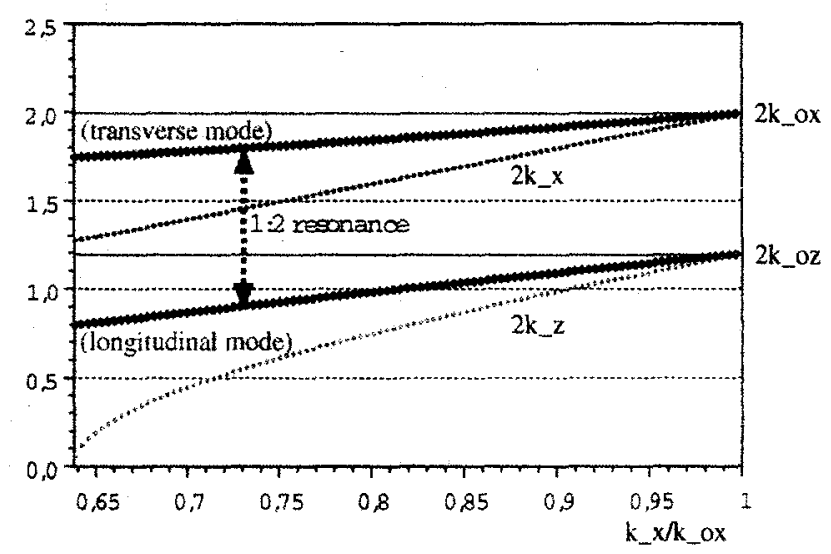

Figure 1: Envelope mode frequencies (normalized to $k_{0, x}$ ) for fixed aspect ratio and zero-current focusing constants as function of tune depression in $\mathrm{x}$.

for vanishing $k_{z}$, which occurs near $k_{x} / k_{0, x}=0.64$, and which corresponds to vanishing longitudinal emittance. 
The point of interest here is that the dispersion of mode frequencies allows a 2:1 coherent resonance condition which enables coupling between the transverse and longitudinal eigenmodes. Obviously, this particular type of coherent resonance condition is lost if $k_{0, z} / k_{0, x}<0.5$.

We can easily explore this coherent resonance behaviour by using the nonlinear envelope equations. For the parameters at the 2:1 resonance condition of Fig. 1, Fig. 2 shows the results based on an initial transverse envelope mismatch, $M M_{x y}=1.5$, and an initial longitudinal mismatch, $M M_{z}=1.0$ (i.e. no initial longitudinal mismatch). For convenience we have arbitrarily chosen $a_{0}=1$. We have defined the initial mismatch factor according to

$$
M M_{x y} \equiv\left(a_{0}+\delta a\right) / a_{0},
$$

where the initial value of the transverse rms envelope is $a_{0}+\delta a$. A similar definition holds for $z$. Later we will have occasion to describe the resulting mismatch observed in an envelope or PIC simulation due to an initial mismatch; we will define the resulting mismatch according to

$$
M M_{x y} \equiv \max \left(a(t) / a_{0}\right),
$$

where $a(t)$ is the rms size, $a_{0}$ is the matched size, and where the maximum is over the duration of the simulation. Returning to Fig. 2, note that there is a resonant exchange

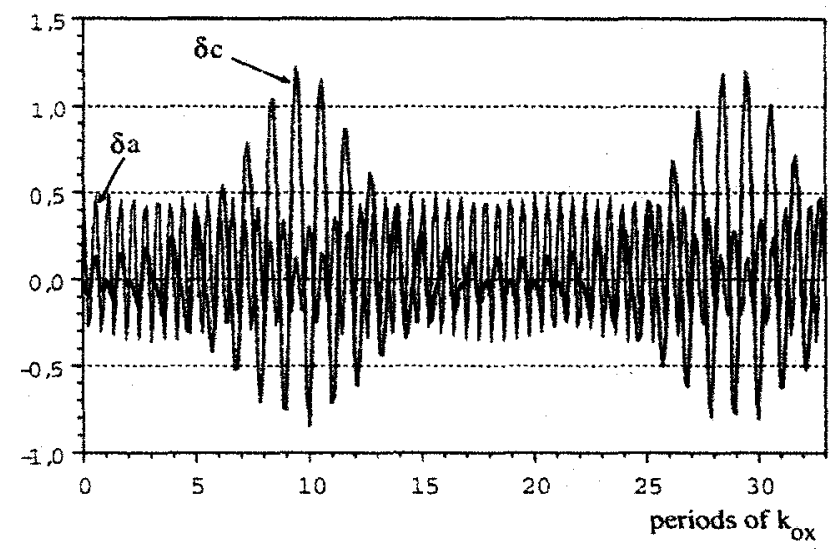

Figure 2: Solutions for $\delta a, \delta c$ from the nonlinear envelope equation at parametric coherent resonance with initial mismatch $M M_{x y}=1.5$ and $a_{0}=1$.

between the transverse and longitudinal mismatch. The latter reaches large amplitude, after which the oscillation energy flows back into the transverse mismatch and the picture repeats. The fact that $\delta c$ exceeds $\delta a$ is attributed to the weaker axial focusing.

A more complete picture of the maximum axial mismatch factors induced by this coupling process is shown in Fig. 3 for fixed $k_{0, z} / k_{0, x}=0.6, k_{x} / k_{0, x}=0.7$, initial $M M_{x y}=1.5$, and the aspect ratio $c_{0} / a_{0}$ as free parameter. There is a stopband for the coupling process around the linear theory parametric resonance condition $\omega_{t o} / \omega_{t r}=0.5$. The width of the stopband is about $\pm 10 \%$ in this frequency

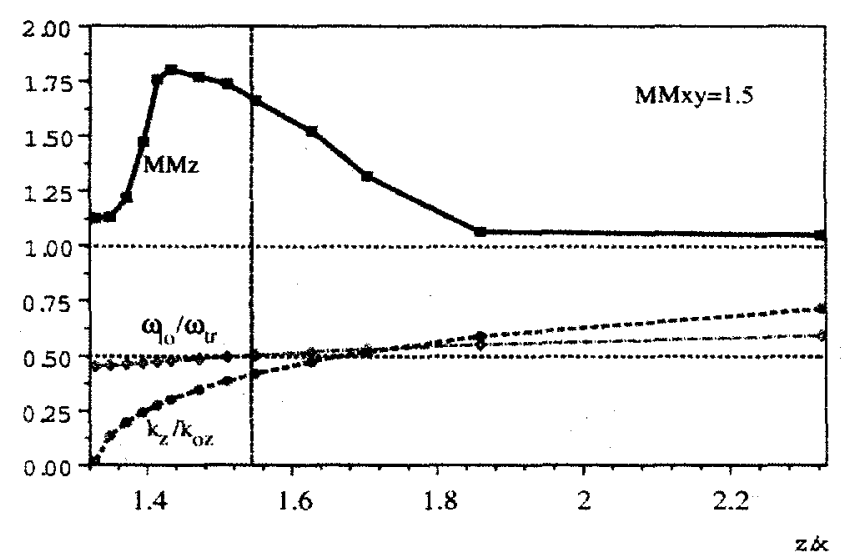

Figure 3: Maximum axial mismatch factors induced by coupling in nonlinear envelope model for fixed $k_{0, z} / k_{0, x}=$ $0.6, k_{x} / k_{0, x}=0.7$ and $M M_{x y}=1.5$ as function of $c_{0} / a_{0}$.

ratio. The maximum longitudinal amplitude is, however, slightly shifted towards smaller frequency ratios, a nonlinear effect that is reduced with decreasing mismatch.

While the 2:1 resonance can provide mismatch coupling from the transverse to the longitudinal direction, the opposite is also possible in terms of a 1:2 resonance. This direction of coupling is, however, found to be less efficient.

\section{3D SIMULATIONS}

This study used a parallel PIC code called IMPACT which was developed as part of a DOE Grand Challenge in Computational Accelerator Physics [5]. For this study we used a second-order accurate, split-operator integration algorithm which involves solving Poisson's equation once in the middle of each step. This is accomplished using standard PIC techniques, namely: (1) depositing charge on a numerical grid, (2) solving Poisson's equation on the grid and obtaining the associated self-field, and (3) interpolating the field from the grid to the particle's positions. The free-space potential is found by convolving the charge density with the Green's function using FFT's and the method of Hockney to analytically move the boundaries to infinity [6].

We present calculations for parameters associated with Fig. 3. We have chosen an initial $6 \mathrm{D}$ waterbag distribution matched by means of the rms equations. The simulation was run for a total of 160 units of time using 4000 integration steps and 20 million simulation particles. At each step a $128^{3}$ grid was placed around the particles for the space charge calculation (though the code actually used an augmented $256^{3}$ grid to solve the free space Poisson equation). To ensure that the bunch was sufficiently stationary, we tracked the initial distribution for 60 units of time prior to applying the envelope mismatch. (In our units, one unit of "time" equals one period of zero current transverse oscillation, i.e. one period corresponding to $k_{o, x}$ ). We ran 4 cases corresponding to 4 different points in Fig. 3 : the exact linearized theory resonance condition (case $\mathrm{A} 1$, 
$\left.\omega_{l o} / \omega_{t r}=0.5\right)$; the point of maximum envelope coupling for the value of $M M x y=1.5$ (case A2); and points at the upper and lower edge of the stopband where practically no coupling is expected (cases A3 and A4, respectively). After applying the mismatch at $t=60$ we observe the coupling and compare results with the envelope model. See Table 1. For cases A1 and A2 the mismatch observed in the simulations is quite close to that predicted by the rms equations, and the results are in qualitative agreement for case A3. Since the development of density inhomogeneity and halo are beyond the envelope model, and since the rms emittances change in the simulations following the mismatch, we do not expect precise agreement.

\begin{tabular}{|c|c|c|c|c|}
\hline case & $\mathrm{A} 1$ & $\mathrm{~A} 2$ & $\mathrm{~A} 3$ & $\mathrm{~A} 4$ \\
\hline$k_{z} / k_{0, z}$ & 0.42 & 0.33 & 0.60 & 0.19 \\
\hline$k_{z} / k_{x}$ & 0.36 & 0.28 & 0.51 & 0.16 \\
\hline$c / a$ & 1.55 & 1.46 & 1.88 & 1.37 \\
\hline$\omega_{l o} / \omega_{t r}$ & 0.50 & 0.48 & 0.55 & 0.46 \\
\hline$M M_{x y}^{\text {envelope }}$ & 1.50 & 1.50 & 1.50 & 1.50 \\
\hline$M M_{z y}^{\text {simulation }}$ & 1.50 & 1.48 & 1.48 & 1.4 \\
\hline$M M_{z}^{\text {envelope }}$ & 1.79 & 1.95 & 1.06 & 1.12 \\
\hline$M M_{z}^{\text {simulation }}$ & 1.76 & 1.87 & 1.25 & 1.6 \\
\hline$X_{\max } / a_{0}$ & 6.6 & 6.5 & 6.1 & 5.9 \\
\hline$Z_{\max } / c_{0}$ & 5.3 & 5.4 & 3.7 & 4.9 \\
\hline
\end{tabular}

Table 1: Summary of 3D rms envelope results and 3D PIC simulation results for $k_{0, z} / k_{0, x}=0.6$ and $k_{x} / k_{0, x}=0.7$.

Besides showing that the coupling predicted in the rms equations is also present in PIC simulations, these results indicate that rms-matched, non-equipartitioned beams in certain parameter regimes may propagate stably for long periods with little-or-no change in the rms quantities. Fig. 4 shows the rms emittances for case Al. Prior to the mismatch at $z=60$ there is almost no emittance growth; the variation is roughly $\pm 0.3 \%$ for the transverse emittances and $\pm 0.7 \%$ for the longitudinal emittance. Fig. 5 shows the

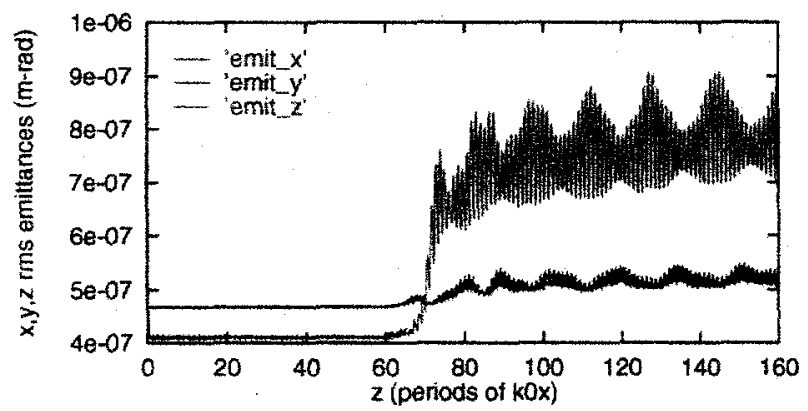

Figure 4: rms emittances vs. $z$ for case A1

rms beam sizes for case Al. There is obvious coupling between the horizontal and transverse planes, with the transverse size reaching a minimum when the longitudinal size is maximum, and vice-versa. By comparison, the corresponding figure for case A3, Fig. 6, shows less coupling.

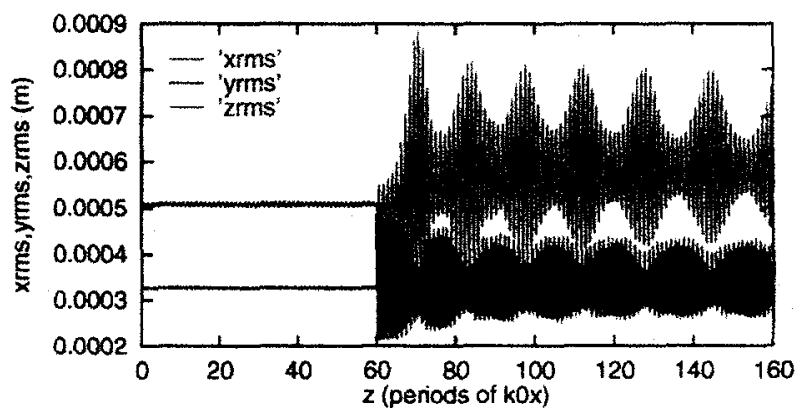

Figure 5: rms beam sizes vs. $z$ for case $\mathrm{Al}$

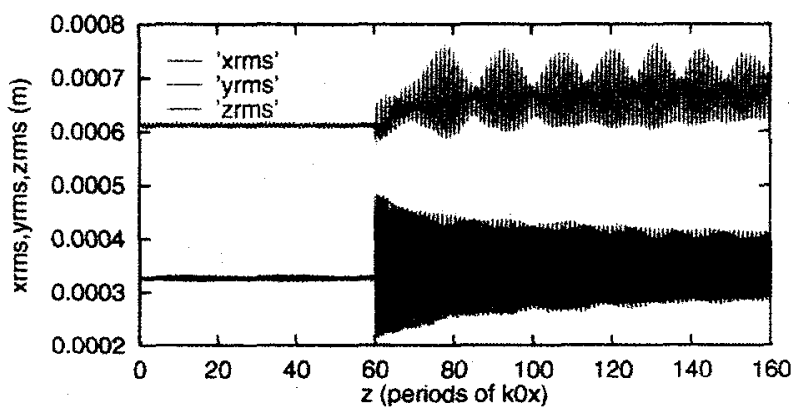

Figure 6: rms beam sizes vs. $z$ for case A3

Case A4 is in fact unstable; we observed significant changes in the rms emittances shortly after the start of the simulation. Note that the initial temperature anisotropy given by $\frac{T_{z}}{T_{z}}=\frac{c k_{z}}{a k_{x}}$ results in a value of 0.56 for the case at resonance (A1), 0.37 for the case of maximum coupling (A2), 0.95 (close to equipartitioned) for case $A 3$, and 0.23 for case A4. The stability of the beams in cases A1, A2, and $\mathrm{A} 3$, and the instability of case $\mathrm{A} 4$, confirms the findings of analytical work in Ref. [3].

\section{ACKNOWLEDGEMENTS}

One of the authors (IH) thanks Los Alamos National Laboratory for its hospitality during recent visits. This research used resources of the National Energy Research Scientific Computing Center, which is supported by the Office of Science of the U.S. Department of Energy under Contract No. DE-AC03-76SF00098. This research also used resources of the Advanced Computing Laboratory located at LANL, which is supported by the U.S. Department of Energy.

\section{REFERENCES}

[1] R.L. Gluckstern et al., Phys. Rev. E, 58, 4977 (1998)

[2] N. Brown and M. Reiser, Phys. Plasmas 2, 965 (1995)

[3] I. Hofmann, Phys. Rev. E, 57, 4713 (1998)

[4] J.S. O'Connell. T.P. Wangler, R.S. Mills and K.R. Crandall, Proc. 1993 Part. Accel. Conf., Washington DC, 3651 (1993)

[5] R. Ryne et al.. Proc. 1998 Linac Conf., Chicago, IL (1998)

[6] R.W. Hockney and J.W. Eastwood, Computer Simulation Using Particles, (Adam Hilger, NY. 1988) 